\title{
LA TEORÍA EPISTÉMICA LAICA Y SU ENFOQUE COGNITIVO-MOTIVACIONAL DEL CONOCIMIENTO
}

\author{
LUIS CARLOS JAUME \\ https://orcid.org/0000-0002-3700-5812 \\ Instituto de Investigaciones de la Facultad de Psicología, UBA-CONICET \\ Universidad de Buenos Aires, Facultad de Psicología, Buenos Aires, Argentina \\ Marcelo Agustín Roca \\ https://orcid.org/0000-0001-8539-2901 \\ Universidad de Buenos Aires, Facultad de Psicología, Buenos Aires, Argentina \\ MANuel TOMÁs PASSERO \\ https://orcid.org/0000-0003-1823-7148 \\ Universidad de Buenos Aires, Facultad de Psicología, Buenos Aires, Argentina \\ SUSANA AZZOLLINI \\ https://orcid.org/0000-0002-3192-5087 \\ Instituto de Investigaciones de la Facultad de Psicología, UBA-CONICET \\ Universidad de Buenos Aires, Facultad de Psicología, Buenos Aires, Argentina
}

Correo electrónico: luiscarlosjaume@gmail.com

Recibido: 15 de septiembre del 2021 / Aceptado: 30 de septiembre del 2021

doi: https://doi.org/10.26439/persona2021.n024(2).5438

\begin{abstract}
RESUMEN. La teoría epistémica laica ha brindado diversos aportes en los campos de la epistemología y de la cognición social, entre ellos, un marco epistémico influido tanto por factores cognitivos como motivacionales. Entre los factores motivacionales se encuentran los conceptos de apertura a nuevas experiencias e impermeabilidad de explicaciones alternativas, los cuales son englobados por Webster y Kruglanski en el constructo de necesidad de cierre cognitivo. Este constructo explica tanto los aspectos motivacionales de la toma de decisiones como la interfaz entre las propiedades cognitivas y motivacionales del individuo en el proceso de adquisición de conocimiento y de creencias. Además, la propuesta epistémica laica abarca también el estudio de los procesos atribucionales y los efectos de las interacciones sociales en las actividades epistémicas. El presente trabajo tiene como propósito el análisis teórico del modelo epistémico laico y su importancia en la epistemología al determinar no solo los aspectos cognitivos, sino también motivacionales del individuo, de los cuales deriva el conocimiento.
\end{abstract}

Palabras clave: epistemología / teoría epistémica laica / necesidad de cierre cognitivo 


\section{LAY EPISTEMIC THEORY AND ITS COGNITIVE-MOTIVATIONAL APPROACH TO KNOWLEDGE}

ABSTRACT. Lay epistemic theory has offered several contributions to the field of both epistemology and social cognition. This theory offers an epistemic framework, which is influenced by both cognitive and motivational factors. Within the motivational factors are the concepts of openness to new experiences and imperviousness to alternative explanations, which are encompassed by Webster and Kruglanski in the construct of need for cognitive closure. This construct explains both the motivational aspects of decision making and the interface between the cognitive and motivational properties of the individual in the process of acquiring knowledge and beliefs. In addition, the lay epistemic approach also encompasses the study of attributional processes and the effects of social interactions on epistemic activities. The purpose of this paper is the theoretical analysis of the lay epistemic model and its importance in epistemology by determining not only the cognitive aspects, but also the motivational aspects of the individual, on which knowledge is derived.

Keywords: epistemology / lay epistemic theory / need for cognitive closure 


\section{INTRODUCCIÓN}

A lo largo de nuestra historia filogenética, los organismos vivos, al estar en contacto con el medioambiente, fueron aprendiendo nuevas funciones que les permitieron una mejor adaptación y, en consecuencia, tener una mayor descendencia (Labrador Montero, 2019). Desde ese entonces hasta hoy en día, los distintos organismos captan la información del mundo exterior para luego convertirla a fin de maximizar la adaptabilidad. Los seres humanos captamos información del exterior y la aprehensión subjetiva de esta genera conocimiento (Juárez \& Salinas, 2012). Es así como la información se transforma en conocimiento; sin embargo, el problema de cómo surge este conocimiento es un debate que aún sigue vigente (López-Silva, 2013; Guerra Osorno, 2019).

Para saciar esta inquietud, la epistemología se ha encargado de plantear una teoría sobre qué es el conocimiento y cómo se construye. Esta rama de la filosofía tiene como propósito el análisis del conocimiento científico por medio del discernimiento de los objetos de estudio planteados por las ciencias, así como los procedimientos lógicos y metodológicos para derivar a determinados estados del conocimiento y la explicación de resultados (Briones, 1996). En el campo epistemológico, existen tres grandes preocupaciones interrelacionadas. La primera es entender el conocimiento. La segunda consiste en estudiar los procesos que utilizamos para producir creencias y/o los distintos métodos de investigación para determinar cuáles de dichos procesos o métodos son conducentes al conocimiento. La última se refiere a elaborar respuestas a los argumentos escépticos que niegan que tenemos conocimiento (Stich, 1990).

Como se dijo anteriormente, los problemas inherentes al conocimiento son cuestiones que continúan vigentes en la actualidad. Esto influye en múltiples dominios de las ciencias sociales y, en particular, en la psicología.

\section{LA COGNICIÓN SOCIAL Y LA CONSTRUCCIÓN DEL CONOCIMIENTO}

La psicología es una disciplina científica que nace a finales del siglo XIX en el laboratorio de psicología experimental de Wilhelm Wundt. Esta ciencia se caracteriza por estudiar y analizar, a través de sus diferentes ramas, la conducta y los procesos mentales tanto de los individuos como de grupos humanos (Cacioppo \& Freberg, 2018; López-Silva, 2013).

Los procesos mentales generados durante la interacción social son estudiados por la cognición social, que es una rama de la psicología. Diversos autores postulan que la cognición social se caracteriza por los procesos subyacentes en las interacciones sociales, como la percepción, interpretación y generación de respuestas ante las interacciones (Ruiz-Ruiz et al., 2006). Los estudios de cognición social permiten explicar los contextos o realidades por las cuales las personas construyen el conocimiento de otros y de sí mismas. Una de las teorías más representativas de las últimas décadas 
sobre los individuos que participan activamente en la construcción del conocimiento es la teoría epistémica laica producida por Kruglanski $(1980,1990)$, la cual explica cómo el conocimiento es un proceso de producción en el que influyen factores cognitivos y motivacionales. Dicha práctica ha tomado como base diversas posturas epistemológicas.

\section{ANTECEDENTES A LA TEORÍA EPISTÉMICA LAICA}

En la década de los setenta hubo un auge de los estudios sobre el enfoque atribucionista a las conductas de las personas. La atribución se refiere a toda actividad epistémica que realizan los sujetos al intentar entender por qué las personas actúan de la forma en que lo hacen $y$, a su vez, el porqué de las conductas propias (Balabanian \& Lemos, 2020). Los estudios relacionados con la atribución de motivos han sido desarrollados por la teoría de la atribución, que intenta explicar los motivos que los individuos dan a las conductas de personas ajenas a ellos mismos (Kelley \& Michela, 1980). Los enfoques atribucionistas proponen que, cuando los sujetos intentan explicar las conductas, utilizan criterios de causas externas o internas (Balabanian \& Lemos, 2020; Heider, 1946; Kelley \& Michela, 1980). Esto concuerda, por ejemplo, con los resultados del experimento de Thibaut y Riecken (1955), donde el factor "clase social" influye en la percepción de la atribución de motivos de los individuos; de este modo, los sujetos de clases más altas son atribuidos con conductas más internas que personas de clases más bajas. Otra conclusión procedente de los mismos autores es que los sujetos tienden a valorar de una forma más positiva a los individuos que están atribuidos internamente que a sujetos con atribuciones externas.

El modelo de ANOVA de Kelley $(1967,1973)$, el cual se encuentra dentro de la teoría atribucionista, propone el concepto de covariación que estipula una relación de causaefecto entre dos variables. Para que se produzca una relación causal, son necesarios cuatro requisitos: (a) distintividad, la cual indica que la atribución causal solo se realizará en presencia de la entidad externa; (b) consistencia en el tiempo, que refiere que la reacción frente a la entidad externa no menguará ni diferirá, siempre y cuando esté presente; (c) consistencia sobre la modalidad, que estipula que la reacción será coherente con la modalidad aunque su interpretación puede diferir; y (d) consenso, es decir que los atributos externos van a ser experimentados de la misma forma por los observadores. Además, el modelo de ANOVA plantea dos propiedades invariantes en el proceso de atribución. Por un lado, una organización temporal relativa a la causa del efecto, ya que el ordenamiento garantiza y refuerza la base implicacional de la lógica en el conocimiento, en el que la presencia adicional de la información condiciona dicho vínculo causal (Kruglanski et al., 2018). Y, por otro lado, el consenso, que permite la aproximación al proceso atribucional sobre la base de una idea compartida acerca del concepto de causalidad (Kruglanski, Hamel et al., 2018). Los individuos que atribuyen el consenso reducen la carga cognitiva y confían en la deducción del grupo. Es decir, la representación 
compartida influye en la atribución de los sujetos a los comportamientos (Kelley, 1967; Zaleskiewicz \& Gasiorowska, 2018).

A diferencia de muchos de los supuestos epistemológicos inherentes a la teoría de la atribución que se fundamentan en la obra de Kelley (1967), Kruglanski (1989), por medio de su teoría epistémica laica, ha sugerido un enfoque teórico alternativo a dicha epistemología, el cual propone sustituir los motivos endógenos (aquellos fines que son un fin en sí mismos) y exógenos (la acción en el medio para obtener otro fin) por una relación de proceso-contenido en la explicación de atribución causal. Por proceso, Kruglanski (1989) se refiere a los aspectos invariantes cognitivos, mecanismos por los cuales todas las personas construyen el conocimiento; mientras que por contenido entiende el aspecto que va a variar dependiendo del grado de relevancia que adquieran ciertos tipos de contenidos de información y su accesibilidad.

\section{LA TEORÍA EPISTÉMICA LAICA}

Según Kruglanski (1990), la teoría epistémica laica debe su término no a una ruptura con la epistemología científica, sino, al contrario, a la comprensión de que tanto el sentido común como el de las ciencias son necesarios. De este modo, la teoría abarca el proceso de adquisición de conocimiento general, que se manifiesta de igual manera en las actividades tanto de los científicos como de los legos (Kruglanski, 1989). El énfasis en dicha epistemología está puesto en lo que tienen en común: las propiedades psicológicas y los modos de adquisición de conocimientos. El proceso de formulación de conocimiento posee mecanismos similares generales, independientemente de qué instituciones o individuos lo estén formulando. Es decir, tanto el conocimiento científico como el no científico comparten características comunes sobre cómo se construye el conocimiento (Kruglanski et al., 2010).

La modificación que realiza el enfoque epistémico laico enfatiza en los criterios de atribución que remiten a los patrones de información que construyen las inferencias. Este mecanismo invariante es lo que genera una consistencia lógica en los procesos inferenciales funcionando como un heurístico, por el que los sujetos utilizan estas reglas inferenciales para relacionar un evento $A$ con un determinado consecuente para la acción A. Esta forma de asociar eventos a lo largo de la filogenética humana ha sido una manera de tratar de maximizar conductas que nos aproximen a eventos recompensantes y evitar eventos negativos que impliquen un riesgo adaptativo. La asunción general de la teoría epistémica es la relevancia que adquiere el mundo exterior, la evidencia y cómo esta es influida por estructuras cognitivas que codifican, modifican y asocian entre síla evidencia. No obstante, los aspectos cognitivos no son el único factor que está relacionado con el proceso de construcción de conocimiento; existen, además, aspectos motivacionales que intervienen en el conocimiento. En este sentido, los sujetos están bajo una motivación 
subyacente de preferir contenidos informacionales completos que les permitan tener una ilusión de control sobre el ambiente (Kruglanski, 1990).

\section{Los factores cognitivos}

La construcción de conocimiento, para Kruglanski (1990), se debe a dos mecanismos que participan en simultáneo: la generación de hipótesis y la validación de hipótesis. Si bien los modelos de generación y validación de hipótesis se han empleado anteriormente para representar las actividades epistémicas en niveles de percepción (Bruner, 1951, 1973; Gregory, 1970, 1973), formación de conceptos (Levine, 1975), resolución de problemas (Bourne et al., 1979; Newell \& Simon, 1972) y descubrimiento científico (Popper, 1972); la teoría epistémica laica (Kruglanski, 1990) hace algunas suposiciones únicas sobre las formas en que se llevan a cabo las funciones de validación y generación de hipótesis. Para Kruglanski (1990), no está del todo claro cómo surge una determinada configuración conceptual, es decir, una hipótesis, a partir de la multiplicidad de conceptos e ideas que fluyen por nuestra mente. No obstante, según el autor, es posible asumir por medio de dos metáforas la conceptualización del mecanismo por el cual los individuos disponen, almacenan y realizan sus actividades epistémicas en la cotidianidad. La primera (la metáfora de los bloques de Lego) hace referencia al conocimiento disponible en nuestra memoria de largo plazo; y la segunda (la metáfora del reflector) aplica a nuestro conocimiento más incipiente, es decir, a nuestra memoria de corto plazo.

\section{La metáfora de los bloques de Lego}

La metáfora de los bloques de Lego trata de la diversidad de cosas que podemos saber (Kruglanski, 1990). Así como los bloques se configuran unos con otros para formar una figura, nuestros conceptos almacenados hacen lo mismo. En otras palabras, se combinan para generar una variedad de nuevos conceptos. Esto nos sugiere que a priori ninguna configuración sería imposible. Según Kruglanski $(1989,1990)$, la relevancia y disponibilidad configurativa de cada bloque depende en gran parte de nuestra predisposición a saber usarlo (o de nuestra compulsión a tener que usarlo). Por otro lado, la relatividad cultural hace su trabajo vehiculizando de manera más fácil ciertos conceptos, es decir, ciertos conocimientos.

Así como una construcción de bloques puede reconstruirse para generar algo nuevo, nuestro conocimiento hace lo mismo. Son propensos a modificarse, revisarse o abandonarse. Siguiendo este hilo de desarrollo, podríamos cuestionarnos si algo es malo o bueno, los gustos, los valores preestablecidos y la propia identidad. Esto es, la metáfora de los bloques de Lego propone pensar nuestro conocimiento actual no como algo inmutable e inmodificable, sino abierto a nuevas construcciones y configuraciones conceptuales. Y, así como nuestros conocimientos individuales son maleables a nuevas configuraciones, las grandes comunidades también modifican los suyos. 
La metáfora del reflector

La metáfora del reflector hace referencia a nuestra capacidad de almacenamiento y atención de la información externa y el conocimiento interno (Kruglanski, 1989, 1990). De este modo, así como nuestro día a día es una heterogeneidad de momentos, nuestro reflector cognitivo alumbra relativas zonas de nuestro conocimiento según las situaciones emergentes. Esto quiere decir - como ya se adelantó anteriormente-que nuestro sistema cognitivo es inestable debido al amplio margen de cambios en nuestro centro de atención. Por ejemplo, preparar una torta nos obliga a que nuestro reflector cognitivo alumbre nuestros conocimientos actuales acerca de "cómo preparar una torta", mientras que nuestros conocimientos acerca de "cómo hacer una fogata" quedan, de manera temporal, en las oscuridades de nuestro sistema cognitivo.

La teoría epistémica laica considera que esta inconsistencia en el funcionamiento cognitivo no es necesariamente un aspecto de patología mental (Kruglanski, 1990). Como los bloques de Lego, nuestro funcionamiento cognitivo nos permite amar y odiar a la misma persona en diferentes ocasiones. Estas configuraciones dependen de la accesibilidad mental de nuestros conocimientos momentáneamente iluminados por nuestro reflector (conciencia). Para múltiples ramas de la psicopatología, la ambivalencia afectiva sería un indicio de que algo anda mal; pero, para la teoría epistémica laica, eso significa y refleja nuestra capacidad de atención selectiva y las múltiples configuraciones posibles de nuestros conocimientos actuales (Kruglanski, Jasko et al., 2018).

\section{Los factores motivacionales}

Una de las posiciones innovadoras de la teoría epistemológica laica es que, además de los factores cognitivos anteriormente mencionados, existen factores motivacionales que juegan un rol importante en el conocimiento (DeBacker \& Crowson, 2009). Esta variante motivacional subyacente es la necesidad de cierre cognitivo (NCC; Kruglanski \& Webster, 1996) que puede tener un individuo, y alude al deseo de los sujetos de poseer ciertas creencias firmes sobre un tema evitando registros de ambigüedad (Horcajo et al., 2011; Jaume et al., 2015; Jost et al., 2003; Kruglanski et al., 2009). Los sujetos perciben en determinadas situaciones que realizar un cierre temprano puede inducir a beneficios 0 costos. Por ejemplo, cuando deben tomar decisiones bajo presión de tiempo requieren procesar la mayor cantidad de información en poco tiempo y emitir su juicio. En otras ocasiones, la fatiga mental o factores externos influyen en los sujetos, los cuales evitan procesar la información (Kruglanski, 2004).

\section{EL “QUÉ” MÁS QUE EL “PORQUÉ” DE LAS MOTIVACIONES EPISTÉMICAS}

El mismo punto de origen puede dar lugar a cualquier motivación epistémica, como también cada motivación puede nacer de diversos orígenes. De esta manera, el giro que 
se produce está relacionado con el "qué", como estado final epistémico, más que con el "porqué". Por ejemplo, las preocupaciones sobre la autoestima podrían generar ocasionalmente la producción de una necesidad de cierre. El fin deseado de la motivación epistémica subyacente en la NCC se refiere al deseo de los individuos de fortalecer una respuesta firme a una pregunta específica y una tendencia a la aversión hacia la ambigüedad. Las actitudes que tienden al cierre sesgan elecciones y preferencias de los individuos, y terminan por inducir afectos negativos cuando el cierre se ve amenazado (Kruglanski, 1989; Távara Ramírez, 2018). Los principales orígenes residen en la necesidad de fortalecer la autoestima, así como en la tendencia a sentirse en control y a poseer la capacidad de responder mediante decisiones precisas. Reiterando esto último, parte del estado final en una necesidad de cierre suele ser el congelamiento epistémico de dicho conocimiento alcanzado. El efecto de congelamiento se define como la base de los juicios que se fundan en los conocimientos preexistentes en vez de usar informaciones posteriores. Por otro lado, el fin deseado de la baja necesidad de cierre consiste en evitar un compromiso epistémico definitivo, así como también una considerable permeabilidad a atribuciones alternativas (Kruglanski, 1989, 1996; Távara Ramírez, 2018).

Si bien nuestras estructuras de conocimiento y de accesibilidad predicen un cierto tipo de comportamiento epistémico, se asume que las tendencias motivacionales coexisten situacionalmente (Moyano \& Trujillo, 2018). Un fin deseado puede modificarse por el cambio de situación y de contexto social. Así, la función principal de la motivación en la atribución causal de los hechos, llevada a cabo por la secuencia de generación de hipótesis y contrastación, se modifica según las circunstancias. Este hecho es análogo al de la metáfora del reflector y de los bloques de Lego. Si las motivaciones cambian situacionalmente, estamos autorizados a decir que existen diferentes confianzas subjetivas en distintas esferas y que las fuerzas motivacionales afectan la dirección del procesamiento de dicha información durante las diferentes actividades situacionales (Kruglanski, 1990, 1989).

Una manera de distinguir estos productos que arroja la motivación epistémica subyacente es agruparlos en dos categorías posibles: factores que afectan los contenidos causales y factores que afectan los juicios. La primera categoría se relaciona con las variables de covariación, es decir, aquellas atribuciones basadas en evidencia de causalidad en las cuales participan la coherencia, el consenso y la distintividad. Estos tres aspectos de la covariación se desarrollan a partir de tipos específicos de entidades externas, como las personas, el tiempo o la modalidad del estímulo externo. Esta categoría de atribución causal basada en los contenidos permite generar inferencias deductivas que parten de esos criterios iniciales hacia las entidades externas. Dependiendo de la relación de esos factores y sus posibles relaciones causales, existirá o no un aumento o disminución en la creencia de esa atribución. El aumento de confianza 
en la explicación causal de un hecho específico se relaciona con la noción, ya mencionada, de magnitud causal, que se reafirma frente a las contrafuerzas de esa explicación; mientras que el descuento en la confianza de una hipótesis se asocia con las posibles hipótesis en competencia. Estos son los factores que afectan e influyen en los contenidos de causalidad.

Por su parte, la segunda categoría está direccionada hacia los juicios propiamente dichos, independientemente de los contenidos atribucionales. En este sentido, las posibles e incipientes fuerzas motivacionales pueden ejercer influencia en los juicios no causales, como, por ejemplo, necesidades de autoservicio como la autoestima, el atractivo físico, ser optimista respecto a ciertos eventos, sin que ello afecte ni se relacione con el proceso causal (Kruglanski, 1989, 1990).

\section{MOTIVACIONES EPISTÉMICAS: BÚSQUEDA DE CIERRE VERSUS EVITACIÓN Y ESPECIFICIDAD}

Lo que posibilita nuestra actividad de conocimiento, y lo que es la base de la adquisición del mismo, es la motivación (Kruglanski, 1989; Rodríguez, 2006). Este concepto hace referencia al grado de interés que se tiene sobre un respectivo tema. Ahora bien, existen diferentes tipos de motivación epistémica (véase la tabla 1); por un lado, los relacionados con la búsqueda o la evitación de ciertos saberes, y, por el otro, los que tienen que ver con la especificidad y la inespecificidad de un asunto. La primera distinción se refiere a si el individuo desea un cierre cognitivo sobre un tema o si desea evitar el cierre y mantener la mente abierta. La segunda distinción se vincula con la especificidad o inespecificidad del cierre deseado (tanto evitado como buscado) (DeBacker \& Crowson, 2009; Horcajo et al., 2011; Kruglanski, 1989).

Tabla 1

Tipos de necesidad de cierre cognitivo

\begin{tabular}{lll}
\hline & Evitación & Búsqueda \\
\hline Específico & Necesidad de evitar un cierre específico & Necesidad de cierre específico \\
Inespecífico & Necesidad de evitar un cierre inespecífico & Necesidad de cierre inespecífico \\
\hline
\end{tabular}

Elaboración propia

\section{Necesidad de cierre inespecífico}

La necesidad de cierre inespecífico significa la búsqueda de un conocimiento que proporcione mayor comprensión de la acción y sus posibles consecuencias. Esta necesidad se caracteriza por la vinculación a un tema, es capaz de generarse a través de diversos motivos, es incentivada por contextos y situaciones específicos, y es esencialmente 
imparcial en sus efectos de juicio (Horcajo et al., 2011; Moyano et al., 2013; Webster \& Kruglanski, 1994).

Las personas pueden variar en la disposición de aprender su mundo en términos claros o inespecíficos. Esto se debe a la relativización de las experiencias sociales en los diferentes contextos. Ahora bien, hay dos grandes características para pensar esto: la de origen diverso y la de imparcialidad. Por un lado, la característica de origen diverso refiere a que la dosis de cierre inespecífico puede surgir de fuentes motivacionales heterogéneas, que dependen del interés intrínseco de la persona, pero esos intereses propios suelen estar muy vinculados con la búsqueda o evitación de cierre. Por otro lado, la idea de excitación situacional remarca el poder del contexto en la motivación epistémica. Esta propiedad se puede derivar en dos posibles categorías: la que corresponde a los beneficios de poseer un conocimiento determinado (relacionado con la previsibilidad que ofrece esa dosis), y la que implica "no cerrar un tema de interés", lo que tiene que ver con el interés de procesar más información. La segunda característica propia del cierre inespecífico, que es la imparcialidad, consiste en el deseo imparcial de conocimiento definitivo sobre un tema en particular (Kruglanski, 1989).

\section{Necesidad de cierre específico}

La necesidad de cierre específico tiene que ver con la expectativa de respuestas particulares y ya no imparciales (DeBacker \& Crowson, 2009). Es decir, si la necesidad de un cierre inespecífico promovía la congelación epistémica, la necesidad de un cierre específico promueve tanto la congelación como la descongelación, lo que depende de si el conocimiento actual es congruente con los intereses actuales de uno (Kruglanski \& Webster, 1996). A partir de lo dicho anteriormente, la necesidad de cierre específico genera un sesgo direccional que influye en el proceso epistémico. Por ejemplo, la necesidad de control puede inducir preferencias hacia cierres epistémicos relacionados con el control (Horcajo et al., 2011; Jaume et al., 2015; Kruglanski \& Webster, 1996).

Al igual que el inespecífico, el cierre específico también puede surgir situacionalmente; es decir, el contexto potencia posibles deseos de un juicio, dado que no había asumido tanta importancia como en aquel momento. En otras palabras, lo novedoso de una situación requiere de un contexto previo que signifique eso novedoso para la persona.

\section{Necesidad de evitar el cierre inespecífico}

Otra de las fuerzas motivacionales en el proceso epistémico es la necesidad de evitar el cierre, la cual postula el deseo, en ciertas situaciones, de evitar el compromiso crítico. Una persona puede valorar la falta de cierre en situaciones en las que existen múltiples elementos de información inconsistente. La necesidad de evitar el cierre puede interpretarse como una reducción en la probabilidad de desencadenar un error. En pocas 
palabras, puede significar la preferencia ocasional por evitar el compromiso cognitivo posiblemente por el miedo a la invalidez (Kruglanski, 1989). Existen casos en los que prevalece la ambigüedad optimista por encima de los cierres sombríos. ¿Prefiero saber que estoy siendo engañado? ¿Me interesan los chismes conyugales de mi amigo(a)? La necesidad de evitar el cierre influye en la actividad epistémica de una persona justo donde un cierre estaba en peligro de formarse. En este sentido, la evitación del cierre se relaciona íntimamente con el proceso de descongelamiento epistémico. El error de un cierre puede generar amenazas a la autoestima, infligir conflictos económicos o poner en peligro el bienestar físico. Como se mencionó sobre el origen de las distintas motivaciones epistémicas, estas nacen situacionalmente en un contexto que las define como aversivas o reforzantes (Kruglanski, 1989, 1990).

\section{Necesidad de evitar un cierre específico}

La motivación para evitar un cierre específico puede conducir a la necesidad de buscar el cierre opuesto, aunque no necesariamente. Si bien puede existir una preferencia por el cierre opuesto positivo, se distinguen dos tipos de motivaciones para evitar los cierres específicos: los individuos motivados para evitar el fracaso y los que se esfuerzan por conseguir el éxito. Los que buscan evitar el fracaso están motivados para evitar un cierre específico, el cual sería "haber fallado"; los segundos están motivados por la necesidad de cierre que afirma el "haber tenido éxito". Ahora bien, la necesidad de evitar el cierre específico puede llevar a la afectación de la congelación epistémica y, en otras situaciones, provocar un descongelamiento epistémico. Es decir, la motivación de evitar "haber fallado en el examen" promueve una fuerte necesidad de evitarlo. Como las demás motivaciones epistémicas, esta nace de orígenes y situaciones diversos. Como en nuestro ejemplo, el miedo a ser rechazado puede conducir a que uno evite la creencia de que no es popular (Kruglanski, 1989).

Ahora bien, esta puesta en marcha de la actividad epistémica no está fragmentada, como sí lo fue el esfuerzo por describirlas aisladamente. En la cotidianidad almacenamos diferentes tipos de motivaciones que se entrelazan o se rechazan situacionalmente; es decir, no es una y para siempre, sino que son varias y variantes.

\section{Compatibilidad entre las motivaciones epistémicas}

Las cuatro motivaciones epistémicas son mutuamente compatibles; es decir, una persona puede almacenar diferentes tipos de motivaciones simultáneamente, la búsqueda y la evitación. Se puede desear el cierre cognitivo de un problema de interés y, a la vez, sentir miedo a la invalidez de aquello que le interesa. La compatibilidad llega hasta el punto en que la existencia simultánea de objetivos epistémicos se vuelve incompatible. En ese caso, es posible la producción de un conflicto o tensión. Es lo que normalmente ocurre; 
es atípico tener un objetivo epistémico específico. Lo típico es la convivencia de heterogeneidades de motivaciones epistémicas que menguan en fuerza dependiendo de la situación (Kruglanski, 1989).

\section{Las motivaciones epistémicas comparadas y contrastadas}

En síntesis, las cuatro fuerzas motivacionales desempeñan la función de poner en marcha la actividad epistémica, más allá de que cada una difiere en su producto final. La necesidad de un cierre inespecífico se satisface con cualquier cierre de un tema pertinente; la necesidad de un cierre específico, donde se forma un cierre; y la necesidad de evitar un cierre, donde no se forma un cierre (véase la figura 1).

Figura 1

Fuerzas motivacionales y actividad epistémica

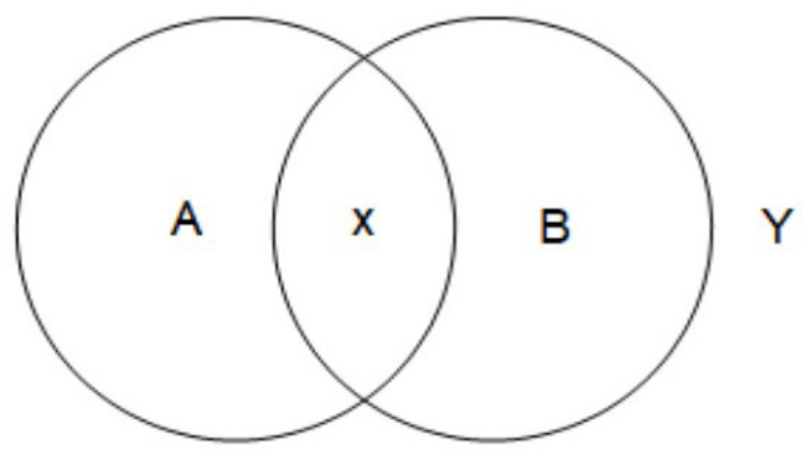

Nota. $\mathrm{A}=$ cierres con propiedad $\mathrm{A} ; \mathrm{X}=$ superposición entre círculos; $\mathrm{B}=$ cierres con propiedad $B ; Y=$ ausencia total de estructuras cognitivas

Elaboración propia

En el círculo $A$, hay un conjunto de cierres con propiedad $A$, mientras que en el círculo $B$ hay un conjunto de cierres con propiedad $B$. Ahora bien, la ausencia de un cierre está representada por la $\mathrm{X}$, por la superposición entre los círculos ( $\mathrm{A}$ y $\mathrm{B}$ ), o por la $\mathrm{Y}$, en la cual hay una ausencia total de estructuras cognitivas (hipótesis) (véase la figura 1). Estas hipótesis, como feto del conocimiento, requieren de un proceso que haga posible su posterior consolidación (validación) o desechamiento (refutación). Este proceso nos sugiere pensar al individuo como portador de una lógica natural, mecanismo incipiente en toda actividad epistémica que promueve la producción del conocimiento (Kruglanski, 1989). Las personas parten de premisas "si..., entonces" a través de las cuales validan sus hipótesis (sus bloques de Lego-conceptuales). Al validar algunas de nuestras ideashipótesis, nuestra confianza se desplaza de nuestra premisa hasta nuestra conclusión. Es decir, todo origen de conocimiento, desde esta teoría, nace de una forma condicional 
que sirve como base para nuestras inferencias deductivas y nuestra actividad epistémica. Si bien las premisas varían según el contexto situacional y la propia biografía del individuo, la lógica legitimadora es la misma: un gurú, un sacerdote, un amigo, un profesor, un sueño, vehiculizan la validación, que es el aspecto uniforme. Es decir, los fenómenos de codificación perceptual pueden regirse por los mismos principios epistémicos que los que gobiernan los niveles conceptuales de adquisición del conocimiento: "si..., entonces", "si es pequeño, tiene mucho pelo y ladra, entonces, es un perro" (Kruglanski, 1989).

\section{CONCLUSIÓN}

A partir de lo desarrollado en el presente trabajo, concluimos que la teoría epistémica laica (Kruglanski, 1989) provee un modelo teórico sobre el cual pensar el proceso de adquisición del conocimiento. Dicho proceso consta de ciertos mecanismos epistémicos subyacentes a toda actividad inherente al ser humano, independientemente de que sean individuos científicos o legos (Kruglanski, 1989). Si bien encontramos diversas explicaciones sobre las fuentes y la naturaleza misma del conocimiento (Briones, 1996; Stich, 1990), Kruglanski (1989) inserta el elemento motivacional como piedra angular de su teoría epistemológica, proponiendo que todo conocimiento parte del grado de motivación presente en el sujeto. Este concepto se encuentra íntimamente ligado al desenvolvimiento de la NCC (Webster \& Kruglanski, 1994), ya que dicho constructo alude al deseo de algunos sujetos de poseer ciertas creencias firmes sobre un tema, evitando registros de ambigüedad (Kruglanski et al., 2009; Jost et al., 2003).

Por último, la teoría propuesta por Kruglanski (1989) genera un marco teórico para el estudio de diversas problemáticas cognitivo-sociales. Es importante que futuras investigaciones logren profundizar en dicha epistemología, ya que no solo es necesario dar cuenta de los procedimientos y técnicas utilizados para comprender las incógnitas, sino también cómo surgen dichos problemas y por qué se producen. La epistemología tiene una gran importancia en los procesos de investigación sociales, dado que nos hace receptores de una capacidad crítica, vinculada no solo al campo de la metodología, sino a los principios mismos de una investigación.

Cómo conocemos y qué nos motiva a ello representa un punto de inicio por parte de la teoría laica para dar mayor consistencia a los nuevos descubrimientos que pueden acaecer en el campo científico.

\section{REFERENCIAS}

Balabanian, C., \& Lemos, V. (2020). El rol de la atribución en el comportamiento prosocial adolescente. Interdisciplinaria. Revista de Psicología y Ciencias Afines, 37(2), 129-142. https://doi.org/10.16888/interd.2020.37.2.8 
Bourne, L. E., Dominowski, R. L., \& Loftus, E. F. (1979). Cognitive Process. Prentice-Hall.

Briones, G. (1996). Epistemología de las ciencias sociales. ICFES.

Bruner, J. S. (1951). Personality Dynamics and the Process of Perceiving. En R. Blake \& G. V. Ramsey (Eds.), Perception: An Approach to Personality (pp. 121-147). Ronald Press.

Bruner, J. S. (1973). Beyond the Information Given: Studies in the Psychology of Knowing. W. W. Norton.

Cacioppo, J. T., \& Freberg, L. (2018). Discovering Psychology: The Science of Mind. Cengage Learning.

DeBacker, T. K., \& Crowson, H. M. (2009). La influencia de la necesidad de cierre en el aprendizaje y la enseñanza. Revisión de Psicología Educativa, 21(4), 303-323. https://doi.org/10.1007/s10648-009-9111-1

Gregory, R. L. (1970). The Intelligent Eye. McGraw-Hill.

Gregory, R. L. (1973). The Confounded Eye. En R. L. Gregory \& E. H. Gombrich (Eds.), Illusions in Nature and Art (pp. 49-96). Duckworth.

Guerra Osorno, I. C. (2019). ¿Una sola epistemología? Interconectando Saberes, 7(4), 1-5. https://doi.org/10.25009/is.v0i7.2620

Heider, F. (1946). Attitudes and Cognitive Organization. Journal of Psychology, 21, 107-112. https://doi.org/10.1080/00223980.1946.9917275

Horcajo, J., Díaz, D., Gandarillas, B., \& Briñol, P. (2011). Adaptación al castellano del Test de Necesidad de Cierre Cognitivo. Psicothema, 23(4), 864-870. http://www. psicothema.com/pdf/3968.pdf

Jaume, L. C., Cervone, N., Biglieri, J., \& Quattrocchi, P. (2015). Propiedades psicométricas del Test Revisado de Necesidad de Cierre Cognitivo (TR-NCC) en una muestra de estudiantes de la Universidad de Buenos Aires. Investigaciones en Psicología, 20(3), 55-60. https://www.psi.uba.ar/investigaciones/revistas/investigaciones/ indice/trabajos_completos/anio20_3/jaume_cervone_biglieri_quattrocchi.pdf

Jost, J. T., Glaser, J., Kruglanski, A. W., \& Sulloway, F. J. (2003). Political Conservatism as Motivated Social Cognition. Psychological Bulletin, 129(3), 339-375. https://doi. org/10.1037/0033-2909.129.3.339

Juárez, J. M., \& Comboni, S. (2012). Epistemología del pensamiento complejo. Reencuentro. Análisis de Problemas Universitarios, (65), 38-51. https://www.redalyc.org/ pdf/340/34024824006.pdf 
Kelley, H. H. (1967). Attribution Theory in Social Psychology. En D. Levine (Ed.), Nebraska Symposium on Motivation (pp. 192-238). University of Nebraska Press.

Kelley, H. H. (1973). Los procesos de atribución causal. Psicólogo Estadounidense, 28(2), 107-128 https://doi.org/10.1037/h0034225

Kelley, H. H., \& Michela, J. L. (1980). Attribution Theory and Research. Annual Review of Psychology, 31(1), 457-501. https://doi.org/10.1146/annurev.ps.31.020180.002325

Kruglanski, A. W. (1980). Lay Epistemic-Logic-Process and Contents: Another Look at Attribution Theory. Psychological Review, 87(1), 70-87. https://doi. org/10.1037/0033-295X.87.1.70

Kruglanski, A. W. (1989). Lay Epistemics and Human Knowledge. Plenum Press.

Kruglanski, A. W. (1990). Lay Epistemic Theory In Social-Cognitive Psychology. Psychological Inquiry, 1(3), 181-197. https://doi.org/10.1207/s15327965pli0103_1

Kruglanski, A. W. (2004). The Quest for the Gist: On Challenges of Going Abstract in Social and Personality Psychology. Personality and Social Psychology Review, 8(2), 156-163. https://doi.org/10.1207/s15327957pspr0802_9

Kruglanski, A. W., Dechesne, M., Orehek, E., \& Pierro, A. (2009). Three Decades of Lay Epistemics: The Why, How, and Who of Knowledge Formation. European Review of Social Psychology, 20(1), 146-191. https://doi.org/10.1080/10463280902860037

Kruglanski, A. W., Hamel, I. Z., Maides, S. A., \& Schwartz, J. M. (2018). Attribution Theory as a Special Case of Lay Epistemology. En J. H. Harvey, W. Ickes y R. F. Kidd (Eds.), New Directions in Attribution Research. Volume 1 (pp. 299-323). Psychology Press.

Kruglanski, A. W., Jasko, K., Chernikova, M., Milyavsky, M., Webber, D., Pierro, A., \& Di Santo, D. (2018). Cognitive Consistency Theory in Social Psychology: A Paradigm Reconsidered. Psychological Inquiry, 29(2), 45-59, https://doi.org/10.1080/1047 840X.2018.1480619

Kruglanski, A. W., Orehek, E., Dechesne, M., \& Pierro, A. (2010). Lay Epistemic Theory: The Motivational, Cognitive, and Social Aspects of Knowledge Formation. Social and Personality Psychology Compass, 4(10), 939-950. https://doi.org/10.1111/j.17519004.2010.00308.x

Kruglanski, A. W., \& Webster, D. M. (1996). Motivated Closing of the Mind: "Seizing" and "Freezing". Psychological Review, 103(2), 263-283. https://doi.org/10.1037/0033295X.103.2.263

Labrador Montero, D. (2019). La evolución de la biología y la biología evolucionista. Especie y finalidad. Revista de Humanidades de Valparaíso, 14, 395-426. http:// dx.doi.org/10.22370/rhv2019iss14pp395-426 
Levine, M. A. (1975). A Cognitive Theory of Learning. Erlbaum.

López-Silva, P. (2013). Realidades, construcciones y dilemas: una revisión filosófica al construccionismo social. Cinta de Moebio, 46, 9-25. https://doi.org/10.4067/ S0717-554X2013000100002

Moyano, M., Expósito, F., \& Trujillo, H. M. (2013). Cierre cognitivo, sexismo y religiosidad: diferencias y similitudes entre grupos de adolescentes con distinta cultura. Anales de Psicología / Annals of Psychology, 29(2), 501-508. https://doi. org/10.6018/analesps.29.2.135601

Moyano, M., \& Trujillo, H. M. (2018). Pérdida de significado, necesidad de cierre cognitivo y extremismo. Ciencia Cognitiva, 12(2), 45-47. http://www.cienciacognitiva.org/ files/2018-4.pdf

Newell, A., \& Simon, H. A. (1972). Human Problem Solving. Prentice-Hall.

Popper, K. R. (1972). Objective Knowledge. Oxford University Press.

Rodríguez, J. O. (2006). La motivación, motor del aprendizaje. Revista Ciencias de la Salud, 4, 158-160. https://revistas.urosario.edu.co/index.php/revsalud/article/ view/548/472

Ruiz-Ruiz, J. C., García-Ferrer, S., \& Fuentes-Durá, I. (2006). La relevancia de la cognición social en la esquizofrenia. Apuntes de Psicología, 24(1-3), 137-155. http://www. apuntesdepsicologia.es/index.php/revista/article/view/74/76

Stich, S. (1990). The Fragmentation of Reason. The MIT Press.

Távara Ramírez, F. A. (2018). Efectos de la saliencia de la mortalidad en la defensa de la visión del mundo y motivos identitarios en católicos y ateos [Tesis de licenciatura, Pontificia Universidad Católica del Perú]. Repositorio digital de tesis y trabajos de investigación PUCP. http://hdl.handle.net/20.500.12404/10158

Thibaut, J. W., \& Riecken, H. W. (1955). Some Determinants and Consequences of the Perception of Social Causality. Journal of Personality, 24(2), 113-133. https://doi. org/10.1111/j.1467-6494.1955.tb01178.x

Webster, D. M., \& Kruglanski, A. W. (1994). Need for Closure Scale. PsycTESTS Dataset. https://doi.org/10.1037/t00995-000

Zaleskiewicz, T., \& Gasiorowska, A. (2018). Tell Me What I Wanted to Hear: Confirmation Effect in Lay Evaluations of Financial Expert Authority. Applied Psychology, 67(4), 686-722. https://doi.org/10.1111/apps.12145 